\title{
Psychodynamique de la honte en prison : les poupées russes de la honte
}

\section{Psychodynamics of shame in prison: The Russian dolls of shame Vincent Combes (Psychologue clinicien) ${ }^{\mathrm{a}, *}$, Sara Skandrani (Maître de conférences en psychopathologie, Psychologue clinicienne) ${ }^{\mathrm{b}}$, François Pommier (Professeur en psychopathologie, Psychiatre, Psychanalyste) ${ }^{c}$}

a UCSA, Maison d'arrêt des Hauts de Seine, 4, rue Marietta-Martin, 75016 Paris, France

b Université Paris Nanterre, EA 4430, AP-HP, Hôpital Cochin, Maison de Solenn, UFR SPSE, 200, avenue de la République, 92000 Nanterre, France

c Université Paris Nanterre, EA 4430, 71, rue de Lancry, 75010 Paris, France

I N F O A R T I C L E

Historique de l'article:

Reçu le 8 novembre 2019

\section{Mots clés :}

Honte

Culpabilité

Prison

Détenu

Délinquance

Criminalité

Humiliation

Stigmatisation

Violence physique

Violence sexuelle

Trauma

\section{R É S U M É}

Objectif. - La honte, bien qu'omniprésente en prison, est souvent cachée ou masquée, enfouie derrière les barreaux. Elle reste peu étudiée par la recherche en psychologie. Nos travaux se sont intéressés à ce sentiment dans le cadre carcéral pour tenter d'en éclairer les visages et les cheminements.

Méthode. - Notre recherche qualitative clinique a été menée dans une maison d'arrêt de la région parisienne, appuyée sur des entretiens de suivi psychothérapeutiques et des groupes de parole sur la parentalité avec des sujets incarcérés.

Résultats. - La honte semble se manifester dès les premiers temps de l'incarcération à travers le corps et les non-dits. Puis elle se dessine plus nettement dans l'histoire des détenus, l'entrée dans la délinquance, ou encore le vécu de l'incarcération. Trois portraits nous permettent d'illustrer différents visages et cheminements de la honte.

\footnotetext{
* Auteur correspondant 
Famille

Groupe de parole

Psychologie légale

Étude qualitative
Transmission

Discussion. - Nous faisons l'hypothèse du caractère central, répétitif et cumulatif de la honte chez les détenus, comme cinq poupées russes emboîtées : la honte germerait dans l'environnement socioculturel et familial ; elle prendrait racine dans la violence et les traumatismes ; elle se révélerait dans la psychodynamique délinquante ; elle serait amplifiée par l'incarcération ; et enfin elle serait transmise en héritage à la génération suivante.

Conclusion. - Cette recherche confirme l'importance structurelle de la honte chez les détenus, déterminant majeur dans leur parcours et semble requérir une approche psychothérapeutique spécifique.

\section{A B S T R A C T}

\section{Keywords:}

Shame

Guilt

Criminality

Jail

Inmate

Humiliation

Discrimination

Physical violence

Sexual violence

Trauma

Support group

Family

Transmission

Forensic psychology

Qualitative study
Objectives. - Shame is omnipresent in jail, often in the shadows or hidden behind the masks of other affects. However, psychological research in a forensic context has paid little attention to this feeling. Method. - This present qualitative study, conducted at a Paris suburban jail, is based on psychotherapeutic interviews and group discussions on parenthood with inmates.

Results. - Shame first reveals itself silently through body language: shifty eyes, sweaty palms, stooped shoulders. Then it becomes more apparent when incarcerated individuals expose their personal background, the origin of their delinquent behavior, or their everyday life in prison.

Discussion. - We posit that shame is structural and cumulative in the life of incarcerated individuals, nested like five Russian dolls in their psyche: first, shame germinates in their social and cultural environment; secondly, it roots itself in the experience of violence and trauma; then it is revealed at the heart of the psychodynamics of crime; it is amplified by imprisonment; and lastly, while still in jail, shame is transmitted to the next generation.

Conclusion. - Our research has demonstrated the prevalence of shame in the psychodynamics of inmates. Further research could focus on defining specific psychotherapeutic approaches that can help them overcome shame.

Puis il descendit seul sous cette voûte sombre. Quand il se fut assis sur sa chaise dans l'ombre Et qu'on eut sur son front fermé le souterrain, L'œil était dans la tombe et regardait Caïn. Victor Hugo, La conscience, La légende des siècles (1859-1877)

This too I know - and wise it were If each could know the same That every prison that men build Is built with bricks of shame, And bound with bars lest Christ should see How men their brothers maim. Oscar Wilde, The Ballad of Reading Gaol (1901) 
Il a fallu me mettre nu

Et quelle voix sinistre ulule

Guillaume qu'es-tu devenu

Guillaume Apollinaire, Alcools (1913)

\section{Introduction}

La honte semble omniprésente dans l'univers carcéral. " Honte pour notre République », clamait le Président Sarkozy au sujet de l'état des prisons devant le Congrès le 22 juin 2009. Honte de la société toute entière qui, pour cacher le stigmate carcéral, « relègue les établissements pénitentiaires loin des centres-villes " ([1], p. 109), dans "l'ombre du monde " [2]. Honte des juges, pour qui la condamnation " est comme une honte supplémentaire (qu'ils ont) honte d'imposer aux condamnés » ([3], p. 16). Honte de ceux qui travaillent pour l'administration pénitentiaire, " résultat d'une contamination morale à la fois par leur institution et par ceux qu'elle héberge " ([2], p. 551). Honte des psychiatres et psychologues, suspectés de s'intéresser aux coupables plutôt qu'aux victimes, et dont l'expertise pénale est dévalorisée [4]. Honte des familles embarquées malgré elles dans une " expérience dévastatrice, quand l'identité des acteurs est brisée par le stigmate associé au statut de proche de détenu » [5]. Et honte des détenus, nous allons le voir, rabaissés, humiliés, honnis.

En outre, la honte est le compagnon de route discret mais incontournable d'une constante de l'incarcération, la culpabilité. Qu'il soit prévenu, donc présumé innocent, ou condamné, la culpabilité est le point commun visible et stigmatisant de tout sujet incarcéré dans son parcours judiciaire et pénitentiaire. La honte n'est pas loin, cachée dans l'ombre de la culpabilité, au point d'être souvent confondue avec elle, et jamais mieux définie qu'en négatif par rapport à elle, dans un rapport dialectique entre pôles : Idéal du Moi/Surmoi ; enfouissement/refoulement [6] ; être/agir ; mère archaïque/loi du père [7] ; désocialisation/socialisation [8] ; fatalité/transgression ; relation narcissique/relation objectale [9].

Omniprésente, la honte en prison est cependant rarement évoquée. La recherche en psychanalyse s'y est d'ailleurs peu intéressée. En cela, le monde carcéral ne fait pas exception, la honte ayant longtemps été le point aveugle de la psychanalyse [10], voire pour certains diamétralement opposées dans leurs logiques : impossible à dire pour la première, misant sur l'effet cathartique de la parole pour la seconde [11].

Depuis quelques années, les recherches témoignent pourtant d'un intérêt croissant pour la honte, point de passage obligé dans l'analyse de la face cachée d'une société narcissique [12]. Mais à quelques exceptions près [13], l'univers carcéral a échappé à ce regain d'intérêt.

Dès lors, il nous a semblé intéressant de mener une recherche sur la psychodynamique de la honte chez les sujets incarcérés.

La honte peut être définie comme " confusion, trouble, sentiment pénible excité dans l'âme par l'idée de quelque déshonneur qu'on a reçu ou qu'on craint de recevoir ou qu'on aurait seulement à ses propres yeux " [14]. Définition qui souligne les deux dimensions inséparables de la honte, intrapsychique et sociale.

Nous nous intéressons ici à la " grande honte " ou " honte disruptive » [14,15]. D'une intensité supérieure à la " petite honte " ou " honte signal d'alarme ", digue psychique proche de la pudeur [16] qui préserve l'intégrité du Moi, la " grande honte " est susceptible de déborder les défenses du Moi et perdure alors que la situation qui a provoqué la honte est passée, avec un sentiment d'indignité intériorisé.

Après avoir exposé notre méthodologie, nous présenterons différents visages de la honte, qui mettent en lumière son caractère central, répétitif et cumulatif, emboîtée dans la vie des détenus comme cinq poupées russes, avec une forte intrication des dimensions subjectives et collectives. 


\section{Population et méthodes}

Notre recherche a été effectuée entre octobre 2017 et avril 2019 au sein de l'Unité de Consultation et de Soins Ambulatoires de la Maison d'arrêt de Nanterre qui accueille 1100 hommes en détention préventive ou pour des peines de moins de deux ans.

Une recherche qualitative clinique, conduite à partir d'un matériel préexistant, nous est apparue adaptée pour approcher la honte, sentiment que les sujets répugnent à évoquer.

Ce matériel est constitué de deux sources complémentaires :

- une cinquantaine de suivis psychothérapeutiques individuels, mis en place à la demande des détenus, avec des rendez-vous de 45 minutes, toutes les deux semaines, sur une période de quelques semaines à plusieurs mois. Le profil de ces patients est varié : hommes majeurs, de 18 à 70 ans, prévenus ou condamnés, pour tous types d'infractions correctionnelles ou criminelles, avec tous types de profils psychologiques ;

- un groupe de parole sur la parentalité, co-animé avec une autre psychologue, qui réunit, d'octobre à juin, toutes les deux semaines, à leur demande, une dizaine de détenus, pères de famille.

Nous avons travaillé suivant une approche complémentariste [17], psychodynamique, afin de mettre en lumière les caractéristiques intrapsychiques de la honte, et psycho-sociale, compte tenu de la double dimension intrapsychique et intersubjective de ce sentiment, et de la grande intrication des dimensions sociales et psychologiques dans la clinique carcérale.

\section{Résultats : visages et cheminements de la honte}

Une recherche sur la honte commence par une recherche de la honte. C'est que la honte se cache et se masque. Elle est comme un " palimpseste impossible à repérer et qui circule souterrainement » [18] sur lequel viennent s'inscrire d'autres sentiments, plus visibles, plus avouables (culpabilité, dégoût, rage, haine, tristesse, peur, vulnérabilité), et d'autres troubles qui ressortent au premier plan (addiction, comportement violent, paranoïa).

\subsection{Premières manifestations de la honte, par le corps et dans les non-dits}

Le corps offre le premier " faisceau de traits cliniques qui amènent l'analyste à penser la honte " [11], notamment chez les nouveaux arrivants : regards fuyants, épaules rentrées, réticence à tendre une main sale ou moite, propos peu assurés.

Après quelques jours, passé le choc carcéral, l'orgueil tente de reprendre le dessus. Ne serait-ce que par instinct de survie, tant les détenus peuvent être violents envers les plus faibles d'entre eux qui portent leur honte en bandoulière. Tel P., patient alcoolique, le visage tuméfié après avoir été roué de coups en promenade, signant un effondrement narcissique impossible à masquer.

Pour les autres, la honte semble se cacher derrière des faux-selfs de fiers et durs. C'est le règne des musculatures gonflées, des apparences soignées, des t-shirts ou baskets de marque, flambants neufs. Dans le bureau du psychologue, le gel hydroalcoolique et la boîte de mouchoirs sont abondamment utilisés, le premier comme pour se désinfecter de la honte autant que des microbes, la seconde pour essuyer des pleurs et la honte qui pourrait les accompagner aux yeux des autres détenus.

Ces manifestations corporelles nous rappellent que la honte peut être autant une émotion qu'une sensation physique et biologique [19].

La honte peut se manifester aussi dans le silence et les non-dits. Détenus qui refusent de consulter un psychologue, murés dans le silence, pour éviter de se mettre à nu ; ou qui consultent pour obtenir une attestation de suivi pour leur dossier judiciaire, et cachent leur honte derrière un discours factuel, stérile, plein d'une fausse assurance.

Silences et non-dits également envers les familles, notamment les enfants. Les groupes de parole sur la parentalité nous montrent ces pères désireux de défendre l'image idéalisée qu'ont d'eux leurs plus jeunes enfants. Avec deux questions récurrentes, sous-tendues par la honte : faut-il voir ses enfants 
au parloir, au risque d'apparaître déchus ? et faut-il dire aux enfants que leur père est en prison, au risque de perdre toute légitimité et autorité ?

Ainsi D. refuse de voir ses enfants et de leur parler. C'est une déchirure, mais son propre père lui a appris que "l'homme est un lion chez lui » et ne doit pas perdre la face. Il est hors de question que ses enfants le voient ou le sachent humilié en prison. Lui-même a toujours défendu l'image de son père restée glorieuse à ses yeux, quand les autres ne voyaient plus qu'un chômeur, rentrant alcoolisé tous les soirs. Pour éviter l'aveu de sa chute, il préfère disparaître le temps de son incarcération.

\subsection{Les multiples faces de la honte}

Derrière ces premières manifestations simples bien que silencieuses, la clinique carcérale révèle une honte aux multiples visages et aux cheminements plus complexes. Pour éclairer notre propos, nous présenterons trois visages de la honte : la honte dans un clivage interculturel chez S., la honte dans l'" éhontement » chez J., et enfin, la honte entre traumatisme et addiction chez $\mathrm{N}$.

S. a 39 ans, l'apparence soignée. Il est divorcé, père de deux filles de 16 et 13 ans, entrepreneur à la tête de plusieurs sandwicheries. Il est incarcéré pour avoir frappé sa fille aînée à coups de ceinture après l'avoir vue parler avec un garçon à la sortie de l'école.

Des sentiments ambivalents de culpabilité et de rage se partagent le centre du tableau. Culpabilité massive d'un homme qui ne se reconnaît pas dans ce " pétage de plomb disproportionné ", souhaite s'amender avec notre aide et espère le pardon de sa fille. Mais rage aussi d'avoir été envoyé ainsi en prison, humilié. Lui n'a jamais porté plainte contre son père qui pourtant le battait régulièrement, «à coups de poing, de pied, de balai ou de fil électrique » : " c'était la norme dans la cité " précise-t-il.

En arrière-plan, masqués par ces deux sentiments, les traits de la honte nous apparaissent sous la forme d'un clivage du Moi, entre deux formes de paternité.

D'un côté, un père épris de modernité occidentale, branché, proche de ses filles, capable d'autoanalyse et prêt à se confier à un psychologue, honteux d'avoir " pété un câble ". Moins fautif (coupable), puisqu'il reproduit ce qui était culturellement admis pour sa génération dans la cité, qu'indigne (honteux), puisqu'il craint surtout d'être disqualifié, abîmé aux yeux de l'objet.

Et de l'autre côté, un père attaché à la tradition, la morale, la pudeur et l'autorité patriarcale de sa culture d'origine. À ses yeux, le père moderne est corrompu par les valeurs occidentales et lui fait honte : " ça ne se fait pas pour une fille de parler avec un garçon! ". On pourrait « jaser sur les filles de S. » dans la cité. "La réputation, c'est sacré ! ".

Ainsi clivé, pris dans une double contrainte de la honte, une confusion des langues interculturelle [20], le Moi peine à équilibrer les tensions internes, qui semblent évacuées par des passages à l'acte violents.

Cette honte pourrait faire écho à une honte plus ancienne, profondément ancrée, familiale et culturelle. Ainsi, S. se souvient que petit, il avait honte de ses parents quand son père prenait sa mère en moto pour l'accompagner pour une course. "Ça ne se fait pas pour une femme de monter à l'arrière d'une moto ou d'un vélo ".

Au sujet de sa mère, $S$. précise, non sans honte, qu'elle est illettrée, maîtrise mal le français, et demande à ses enfants de remplir les papiers administratifs à sa place.

Son père, figure autoritaire et distante, s'est beaucoup sacrifié pour sa famille. Originaire du Rif marocain, orphelin de père à 9 ans et aîné d'une fratrie de sept enfants, il a dû émigrer à 17 ans pour subvenir aux besoins de sa famille au bled, devant se contenter d'un modeste refuge dans le bidonville de Nanterre. Ayant le sentiment d'avoir tout donné pour sa famille, il s'est montré très exigeant envers ses enfants, punissant violemment tout écart. S. a souvent eu honte de ne pas être à la hauteur des attentes de son père. Mais dans le même temps, S. était excédé par son attitude toujours pleine de déférence vis-à-vis des " français de souche ", comme si son père souffrait d'un sentiment permanent d'indignité, d'infériorité.

Un autre visage de la honte, en négatif cette fois, dans l' " éhontement », est celui de J., 22 ans, incarcéré pour agression sexuelle sur deux jeunes garçons, de 4 et 7 ans, qu'il gardait comme baby-sitter. Sans pudeur aucune, il nous détaille son crime avec abondance de détails scabreux. Se revendiquant comme ayant " des principes, des valeurs, une propension à faire le bien ", à " donner de sa personne " (sic), il dit ne pas avoir perçu le préjudice de ses écarts, n'ayant ni usé de la contrainte physique, ni 
rencontré d'opposition des enfants. Son attirance pour les jeunes garçons est ancienne : il fréquente des sites pédophiles depuis longtemps, mais tente de lutter contre ses pulsions et souhaite se faire soigner.

Un événement douloureux ressort d'une enfance sinon décrite comme heureuse, " enfant unique de parents unis ". Tout petit, J. a dû subir une opération pour une malformation congénitale du tube digestif. Il en a gardé une longue cicatrice verticale, de part et d'autre de son nombril. Il en a toujours été complexé. En soulevant son t-shirt, il nous exhibe son ventre et, appuyant sur les côtés de son abdomen, fait rentrer le nombril et la cicatrice, et ressortir deux bourrelets de part et d'autre de la raie que forme sa cicatrice. Il explique qu'à l'école, dans les vestiaires, on se moquait de son "ventre comme un cul ». Cet exhibitionnisme soudain nous renvoie à l'analité de la honte avec une confusion devant/derrière caractéristique $[6,14]$.

Chez J. la culpabilité affichée nous semble peu sincère et la honte absente. Le contre-transfert négatif provoqué à l'évocation du crime est redoublé par le discours éhonté du patient. " Ehontement " qui peut être appréhendé comme une " contre-honte défensive " [21] ou une formation réactionnelle de la honte [22] : la honte, inconsciente, projetée, échappe au sujet et n'est éprouvée que par l'entourage [6].

N. nous offre un troisième visage de la honte, entre traumatisme et addiction. Âgé de 49 ans, marié, père de deux enfants, professeur de tennis, souriant et agité, $N$. est incarcéré pour tentative de vol et encourt une courte peine. Il n'en dira rien à ses enfants, pour " préserver (son) image à leurs yeux, et la leur aux yeux de leurs copains à l'école », comme pour éviter ainsi la contagion de la honte.

Violé entre 8 et 16 ans par un éducateur de l'association sportive de son quartier, il a tenté de retourner la situation à son avantage en obtenant, par le chantage, des dédommagements financiers et matériels de son agresseur. Il n'en a jamais parlé à personne, se sentant à la fois honteux, craignant " d'être pris pour un homosexuel ", et coupable d'avoir tiré profit de la situation. Suivant Ferenczi [23], on retrouve ici un enfant " clivé, à la fois innocent et coupable ", qui réagit " non par la défense, mais par l'identification anxieuse et l'introjection de celui qui (le) menace ou l'agresse », le " rendant encore plus profondément conscient de sa faute et encore plus honteux".

Comme s'il cherchait à blanchir l'argent sale de la honte, N. s'est montré très généreux autour de lui. Nombreux cadeaux à ses proches ou, comme le $1^{\mathrm{er}}$ mai, quand il achetait 500 brins de muguet pour les femmes de son quartier. Cette défense maniaque pour échapper à une menace d'effondrement narcissique, semble avoir été alimentée par l'addiction : cannabis dès 12 ans, ecstasy en soirée de 18 à 22 ans, cocaïne entre 20 et 35 ans, crack parfois et alcool toujours. Sans oublier le jeu qui faisait de lui un VIP au Casino de Deauville. Il a dealé aussi, mais précise fièrement qu'il ne s'est jamais fait arrêter. Il affirme n'avoir jamais été dépendant, mais avoue un " dérapage " suivi d'un séjour à Sainte-Anne. Avec sa femme, ils ont arrêté de consommer à l'arrivée de leurs enfants, il y a une dizaine d'années.

Donnant le sentiment d'être en quête de reconnaissance, il dit s'être installé " loin de la cité, dans les beaux quartiers ", où il donne des cours de tennis et rêve d'offrir à sa femme un salon de coiffure - spa.

N. n'est pas le seul traumatisé de sa famille : sa femme et sa belle-mère ont également été violées. Quant à sa propre mère, qu'il décrit comme autoritaire et peu affectueuse ( je ne me souviens que d'un seul bisou pendant toute mon enfance "), elle a été témoin de l'assassinat de ses deux parents pendant la guerre d'Algérie, et blessée elle-même de deux balles dans la cuisse. Interrogé sur cet épisode, N. avoue ne pas en savoir plus, le sujet étant tabou dans sa famille.

\section{Discussion : les poupées russes de la honte}

Notre recherche nous amène à avancer l'hypothèse de la répétition et de l'accumulation de la honte [24]. La honte serait emboîtée dans la vie psychique des sujets incarcérés comme cinq poupées russes, avec une forte intrication des dimensions intrapsychiques et intersubjectives :

- la honte germerait dans l'environnement socioculturel et familial ;

- elle s'enracinerait dans l'expérience de la violence et du trauma ;

- elle se manifesterait au cœur de la psychodynamique délinquante ;

- elle serait amplifiée par l'incarcération ;

- enfin, elle serait transmise en héritage à la génération suivante. 


\subsection{La honte germerait dans l'environnement socioculturel et familial}

Quand 70\% des Français jugent qu'il y a trop d'étrangers en France et $74 \%$ que la religion musulmane est incompatible avec la société française [25], les détenus, majoritairement issus du Maghreb et de confession musulmane, ont pu grandir en intériorisant une honte d'eux-mêmes ([26], p. 161).

Une honte à double face : côté pile, c'est la honte des enfants pour leurs parents illettrés, parlant mal le français, pauvres, sans emploi, marginalisés, humiliés. Côté face, c'est la honte des enfants de ne pas être à la hauteur des aspirations de parents qui ont tout sacrifié pour leur famille. On retrouve bien ces deux faces chez $\mathrm{S}$. : crainte de faire honte à son père, honte de sa mère illettrée ou de son père trop déférent.

La honte semble également pouvoir venir d'une transmission intergénérationnelle. Une honte « qui ne se dit pas mais imprime profondément sa trace dans le psychisme, survit et se transmet au fil des générations " [11]. Honte des parents, issus de l'immigration, victimes des traumatismes multiples de l'Histoire (colonisation, guerres d'indépendance, émigration, intégration) qui se sont accompagnées des humiliations, discriminations et stigmatisations, propices à l'émergence de la honte. Ainsi le père de S., qui paraît être en quête permanente de reconnaissance pour sortir d'une chaîne intergénérationnelle de discrimination et de stigmatisation, comme Rifain au Maroc, puis comme Maghrébin en France. Ou encore la mère de $\mathrm{N}$. blessée et témoin de l'assassinat de ses parents pendant la guerre d'Algérie, dont le traumatisme semble ne pas avoir pu être élaboré du fait de la " silenciation " [27] de la petite histoire qui rejoint celle de la grande Histoire. Honte qui resterait ainsi inavouée, inconsciente, enfermée dans une " crypte ", et donnant naissance, à la génération suivante, à des "fantômes " de honte, qui hantent les enfants [28]. " À défaut d'être dits, les traumatismes perdurent de génération en génération quelle que soit leur origine, individuelle, familiale ou collective » ([29], p. 6).

\subsection{La honte s'enracinerait dans l'expérience de la violence et du trauma}

L'expérience de la violence est un des traits communs à nombre de détenus. Or violence et honte apparaissent unies dans une relation de causalité circulaire.

Les violences subies, et les traumatismes qui les accompagnent, semblent omniprésents dans l'anamnèse des sujets écroués. Violences physiques, comme $S$. maltraité par son père, ou sexuelles, comme N. Les victimes de ces violences, « quand elles arrivent à se faire entendre, évoquent toutes le sentiment de honte qui les habite " ([26], p. 119).

Ce sont aussi les violences économiques (pauvreté, chômage, précarité), sociales (ségrégation socioculturelle, inégalités, injustices), symboliques (stigmatisation, humiliation), psychologiques (dévalorisation, rejet affectif), qui s'accompagnent chez les sujets de sentiments de petitesse, de disqualification ou d'invalidation. Ce terrain fertile semble favoriser l'enracinement de la honte.

Quant aux violences commises, violences verbales, physiques ou sexuelles, comme celles de S. ou de J., à l'origine de leur incarcération, elles peuvent apparaître comme un destin pour la honte et les sentiments de dégoût, de rage ou de haine qui les accompagnent [30-32].

\subsection{La honte se révélerait dans la psychodynamique délinquante}

Les recherches en psychocriminologie soulignent la place centrale que peut prendre la honte dans la psychodynamique délinquante [33]. Chez les " criminels d'habitude ", les agirs antisociaux s'expliqueraient par le surinvestissement du Moi Idéal et l'effacement simultané du Surmoi (et de la culpabilité), mis hors-circuit par clivage et projection. Le Moi ferait siennes les aspirations de toutepuissance du Moi Idéal et concentrerait sa lutte défensive contre la honte et les sentiments associés de petitesse, d'infériorité que le criminel trouve insupportables.

Avec l'incarcération, le Surmoi reviendrait toutefois sur le devant de la scène interne, dans sa dimension anti-narcissique, humiliant le Moi qui a échoué dans son projet d'expansion narcissique avec le Moi Idéal. Ce qui éveillerait les sentiments de honte du détenu : " honte de se découvrir tout petit alors même que l'on s'est cru grand, d'être réduit à être un misérable vers de terre, un rebut, une déjection alors qu'on se croyait un homme " [34]. 
$\mathrm{N}$. illustre bien ce conflit entre instances : pour tenter de sortir de la honte du viol et du chantage, en quête d'amour et de reconnaissance, son Moi semble s'allier avec son Moi Idéal, et fait preuve d'ambition et d'une générosité débordante. Le Surmoi, relégué au deuxième plan, paraît laisser la voie libre à la délinquance et à la drogue, renforçant un sentiment de toute-puissance. Jusqu'à ce que l'arrestation vienne marquer l'échec de l'alliance du Moi avec son Idéal, et le retour du Surmoi, et de la honte.

\subsection{La honte serait amplifiée par l'incarcération}

L'effet de l'incarcération sur la honte semble paradoxal. À la différence de l'humiliation publique qui a longtemps fait partie de l'arsenal punitif avec le pilori ou le carcan [3], la prison peut faciliter l'enfouissement, une des principales réactions défensives face à la honte. La détention permettrait au sujet, comme Edipe, de se rendre aveugle pour, ne plus " se voir être vu », " éviter la honte qui surgirait de son propre reflet, de sa propre misère, réverbéré dans/par les yeux (...) des autres » ([35], p. 160).

Mais cet enfouissement de la honte paraît voué à l'échec, à l'image de la vaine tentative de Caïn d'échapper au regard de "l'œil " [36]. De fait, le regard honnisseur semble transpercer les murs, se défier des barreaux et mettre à nu l'âme du détenu dans sa cellule.

Quant au corps, s'il est exempté de la mise en scène moyenâgeuse, il n'en semble pas moins soumis aux affres d'un quotidien éminemment " hontogène " : " prise de corps » humiliante devant la famille, manque de sommeil et d'hygiène des gardes à vue, fouilles au corps intégrales, absence d'intimité quand uriner, excréter, prendre une douche, manger se font sous le regard des autres, les codétenus, les gardiens, les caméras, voire le monde extérieur. À l'exemple de M., qui nous paraît submergé de honte et de rage après qu'un autre détenu l'a filmé sous la douche et posté la vidéo sur Snapchat. Cet online shaming, version contemporaine du pilori, réunit ici la nudité physique et la nudité psychique, et peut s'accompagner de sentiments de perte d'emprise, de passivation, de petitesse, actant le retournement de l'exhibition phallique en exposition anale [37] caractéristique de la honte.

\subsection{La honte serait transmise en héritage à la génération suivante}

Héritiers de la honte, les détenus peuvent, à leur tour, la transmettre à leurs enfants. Les non-dits des pères, pourtant motivés par le désir d'éviter la honte, s'inscrivent dans une possible transmission intergénérationnelle, quand la honte empruntée devient une honte prêtée.

D'une part, " l'enfant intériorise la honte du parent comme une manière de lui rester lié et aussi, souvent, de réaliser imaginairement le désir de le guérir ». " Mais non seulement le fait d'intérioriser le parent honteux ne permet pas de guérir celui-ci de son malaise, mais en plus, l'enfant qui réalise cette opération psychique risque bien de traîner tout au long de sa vie cette honte d'un autre en lui » ([38], p. 56).

Ainsi A. confesse au " groupe pères " avoir expliqué à ses trois enfants de 3,5 et 7 ans qu'il travaillait à l'aéroport. Les deux plus petits n'y ont vu que du feu, les portiques de sécurité étant similaires. Mais c'est plus compliqué avec sa fille de 7 ans qui a compris, mais n'en dit rien. Inquiet, A. note que ses résultats scolaires se détériorent et s'interroge sur son mutisme récent. Elle sait ; A. sait qu'elle sait ; et elle sait que son père sait qu'elle sait. Mais rien n'est dit, le sujet est soigneusement évité, le sceau du secret semble se refermer sur la honte. Quand son petit frère de 5 ans interroge leur père lors d'un parloir sur ses activités à l'aéroport, elle l'interrompt brusquement : " mais laisse donc papa tranquille, tu vas le fatiguer ! », comme si elle cherchait à protéger son père et le secret dans un même mouvement.

\section{Conclusion}

Notre travail de recherche en prison a mis en lumière l'importance de la honte dans le fonctionnement psychique des détenus. La honte en cellule, à l'instar d'une cellule souche en biologie, possède des propriétés d'auto-renouvellement et de différenciation [39].

Avec l'auto-renouvellement, la division d'une cellule souche donne naissance à deux cellules filles identiques à la cellule mère. La honte en cellule semble disposer de cette même propriété de reproduction à l'identique entre générations, empruntée aux parents, prêtée aux enfants. 
La différenciation d'une cellule souche est fonction de l'expression de ses gènes ou des stimuli de son environnement. De façon similaire, en fonction de l'environnement et de la personnalité des sujets, la honte en cellule peut se différencier de multiples façons, en négatif dans l'« éhontement » et simultanément en miroir chez le clinicien, ou encore masquée derrière d'autres sentiments ou troubles.

Quelle que soit l'image retenue, cellule souche ou poupées russes, on voit à quel point la honte semble incrustée chez les sujets incarcérés, avec une grande intrication des dimensions subjectives ou collectives.

Accepter, comprendre et dépasser sa honte n'en paraît que plus compliqué. C'est tout l'enjeu des approches psychothérapiques spécifiques qui misent sur le partage d'affect [6], la clinique transindividuelle [10] ou groupale [26], et qui pourraient faire l'objet de recherches-actions futures.

\section{Déclaration de liens d'intérêts}

Les auteurs déclarent ne pas avoir de liens d’intérêts.

\section{Références}

[1] Milhaud O. Séparer et punir. Une géographie des prisons françaises. Paris: Cnrs; 2017.

[2] Fassin D. L’Ombre du monde. Une anthropologie de la condition carcérale. Paris: Le Seuil; 2015.

[3] Foucault M. Surveiller et punir. Naissance de la prison. Paris: Gallimard; 2016.

[4] Zagury D. L'expertise psychiatrique pénale : une honte française. La Gazette du palais 2016;19:12-5.

[5] Touraut C. La famille à l'épreuve de la prison. Paris: PUF; 2012.

[6] Ciccone A, Ferrant A. Honte, culpabilité et traumatisme. $2^{\mathrm{e}}$ ed. Paris: Dunod; 2015.

[7] Raoult PA, Labrune L. La honte à l'adolescence : de l'affect au lien social. Paris: In Press; 2014.

[8] Tisseron S. De la honte qui tue à la honte qui sauve. Le Coq-Heron 2006;(1):18-31.

[9] Green A. Le narcissisme moral. Narcissisme de vie, narcissisme de mort. Paris: Éditions de Minuit; 1983. p. 177-206.

[10] Tisseron S. La honte : psychanalyse d'un lien social. $3^{\text {e }}$ ed. Paris: Dunod; 2014.

[11] Barazer C. Ulysse nu et couvert de boue. Rev Fr Psychanal 2003;67(5):1789-94.

[12] Shah A. The cultural faces of shame. In: Akhtar S, editor. Shame. Londres: Karnac; 2016. p. 49-70.

[13] Gilligan J. Shame, guilt, and violence. Soc Res 2003;70(4):1149-80.

[14] Janin C. Pour une théorie psychanalytique de la honte (honte originaire, honte des origines, origines de la honte). Rev Fr Psychanal 2003;67(5):1657-742.

[15] Ferrant A. Le regard, la honte et le groupe. Rev Psychother Psychanal Groupe 2004;2:145-58.

[16] Freud S. Trois essais sur la théorie sexuelle. Paris: Gallimard; 1991.

[17] Devereux G. Ethnopsychanalyse complémentariste. Paris: Flammarion; 1972.

[18] Lévy G. Actuelles sur la cruauté et la honte. Le Coq-héron 2006;1:40-7.

[19] Adelman AJ. The analyst's sense of shame. In: Akhtar S, editor. Shame. Londres: Karnac; 2016. p. 185-203.

[20] Akhtar S. Shame and shamelessness. In: Akhtar S, editor. Shame. Londres: Karnac; 2016. p. 93-113.

[21] Orasanu B. Des mots qui n’arrivent pas à voir. In: André J, Beetschen A, Dargent F, Georgescu M, Neau F, Orasanu B, editors. La Honte. Paris: PUF, coll. « Petite bibliothèque de psychanalyse "; 2017.

[22] Wurmser L. Primary shame, mortal wound and tragic circularity: some new reflections on shame and shame conflicts. Int J Psychoanal 2015;96(6):1615-34.

[23] Ferenczi S. Confusion de langues entre les adultes et l'enfant. In: Psychanalyse IV. Euvres complètes, 4, 1927-1933. Paris: Payot; 1982. p. 125-38.

[24] Ernaux A. La honte. Paris: Gallimard; 1997.

[25] Le Bars S, Monde. La religion musulmane fait l'objet d'un profond rejet de la part des Français. Le Monde [en ligne]; 2013 [consulté le 13/01/2020]. Disponible sur : https://www.lemonde.fr/societe/article/2013/01/24/la-religion-musulmanefait-l-objet-d-un-profond-rejet-de-la-part-des-francais_1821698_3224.html.

[26] De Gaulejac V. Les sources de la honte. Paris: Desclée de Brouwer; 2008.

[27] Cherki A. Honte et regard. Le Coq-heron 2017;1:54-63.

[28] Abraham N, Torok M. L'écorce et le noyau. Paris: Flammarion; 1987.

[29] Tisseron S. Les secrets de famille. Paris: PUF, col. « Que sais-je ? »; 2017.

[30] Rushdie S. Shame. Toronto: Vintage Canada; 1995.

[31] Stein R. La honte : métapsychologie et expérience. Rev Fr Psychanal 2003;67(5):1869-73.

[32] Benslama F. Un furieux désir de sacrifice. Le surmusulman. Paris: Le Seuil; 2016.

[33] Brunet L, Casoni D. Culpabilité, honte et dynamique criminelle. Rev Fr Psychanal 2003;67(5):1561-5.

[34] Merot P. La honte : «Si un autre venait à l'apprendre ». Rev Fr Psychanal 2003;67(5):1743-56.

[35] Roussillon R. Les paradoxes et la honte d'Edipe. In: Paradoxes et situations limites de la psychanalyse. Paris: PUF; 2005. p. 7-164.

[36] Hugo V. La Conscience. La légende des siècles, première série. Paris: Gallimard, NRF; 2002.

[37] Chasseguet-Smirgel J. La maladie d'idéalité : essai psychanalytique sur l'idéal du moi. Paris: L'Harmattan; 1999.

[38] Tisseron S. Les secrets de famille, la honte, leurs images et leurs objets. In: La psychanalyse avec Nicolas Abraham et Maria Torok. Paris: Érès; 2001. p. 53-68.

[39] Institut national de la santé et de la recherche médicale (INSERM). MOOC côté cours : Qu'est-ce qu'une cellule souche? [en ligne]. 2015 mai [consulté le 13/01/2020]. Disponible sur : https://www.youtube.com/watch?v=TBvgGrXuqcY. 